\title{
Failure Mode and Effect Analysis of Subsea Multiphase Pump Equipment
}

\author{
Shobowale Kafayat Oluwatoyin ${ }^{1, \text { a }}$, Fakhruldin Mohd Hashim²,b and Hilmi Bin Hussin ${ }^{3, \mathrm{c}}$ \\ ${ }^{123}$ Department of Mechanical Engineering, Universiti Teknologi PETRONAS, Bander Seri Iskander, \\ 31750, Tronoh, Perak, Malaysia
}

\begin{abstract}
Finding oil and gas reserves in deep/harsh environment with challenging reservoir and field conditions, subsea multiphase pumping benefits has found its way to provide solutions to these issues. Challenges such as failure issues that are still surging the industry and with the current practice of information hiding, this issues becomes even more difficult to tackle. Although, there are some joint industry projects which are only accessible to its members, still there is a need to have a clear understanding of these equipment groups so as to know which issues to focus attention on. A failure mode and effect analysis (FMEA) is a potential first aid in understanding this equipment groups. A survey questionnaire/interview was conducted with the oil and gas operating company and equipment manufacturer based on the literature review. The results indicates that these equipment's group are similar with its onshore counterpart, but the difference is the robustness built into the equipment internal subsystems for subsea applications. The results from the manufacturer perspectives indicates that Helico-axial multiphase pump have a mean time to failure of more than 10 years, twin-screw and electrical submersible pumps are still struggling with a mean time to failure of less than 5 years.
\end{abstract}

\section{Introduction}

Oil and gas reserves in shallow water are fast becoming depleted and oil and gas exploration and production companies are exploring the deepwater/subsea reserves which has been characterized as a risky operation largely due to the fact that there is a lot of uncertainties as regards the performance of the equipments owing to fluctuating operating conditions these equipment's will be subjected to. The equipment's that have been deployed subsea includes: Helico-Axial Multiphase Pump (HAP) whose current depth of deployment around 3000 meters and the mean time to failure (MTTF) is around 12 years according to the equipment manufacturer. Twin-Screw Pump (TSP) current depth of deployment is around $1000-1500$ meters. The performance of these TSP's is a function of screw diameter, pitch and speed (Bornemann pump manual) and MTTF of about 2 years while Electrical Submersible Pump

a Corresponding author : shobowale_g01955@utp.edu.my 
(ESP) whose current depth of deployment is around 1500-2000 meters and MTTF of about 3 years. ESP symptom of a failure and the description of the effect by which a failure is observed also includes: Pump intake blockage, increased reservoir pressure, broken pump shaft, hole in tubing which is due to the presence of high water cuts, production of fines, high salinity, and corrosion [1]

Each subsea multiphase pump one has their own individual applicability, operating range and technical challenges [2]. Usually, customized variant of the equipment is what is obtainable in the field, the behavior and experience achieved with these types of configuration can be used as inference for the important components part that should be taken into consideration for the assessment of this equipment.

Some failure modes and effects from the literature includes work of [3], who performed FMEA based on literature review and came up with a list of important components that can cause failure in helico-axial multiphase pump deployed in subsea environments based on one failure mode which was loss of head/pressure of the pump. [1,4,5] also lists some typical failure effects that can be attributed to electrical submersible pumps in subsea field, [6,7,8] for helico-axial multiphase pump and $[6,9,10,11,12]$ for Twin-Screw Pump.

\section{Research Methodology}

A questionnaire was designed to elicit the failure mode and effect of each equipment group of subsea multiphase pumping equipment from the experts. The questionnaire was designed based on literature review and discussion with experts in the oil and gas industry. A total 30 questionnaires were prepared to be administered during the Asian Oil, Gas and Petrochemical Engineering Exhibition (OGA 2013) exhibition held in Kuala Lumpur, Malaysia, in June, 2013 but only seven respondents participated in the questionnaire survey. Even though, more than 1000 companies from over 50 countries participated in the exhibition, only very few has the require knowledge and skills to provide answers to the questionnaire. Most of respondent's are knowledgeable about the equipment being deployed onshore but not subsea. The rest of the questionnaire was administered through e-mail follow up and interview with the oil and gas company and equipment manufacturer. The interview items shared with the experts includes: a generic prototype schematic drawing of each equipment which was adjusted by the experts and the FMEA worksheet. Typical question from the questinnaire includes: "For Helico-axial multiphase pump for use in subsea environment, Please indicate for each component the parameter that affects its functionality, the cause, the effects, the measures and recommendations"

\section{Results and Discussion}

According to the analysis of the respondent profile, the only expert having 20 years and above (Table 1) working experience also has a $\mathrm{PhD}$ degree (Table 2) and a manager (Table 3) in an engineering contractor company (Table 4) that supplies all the subsea processing equipment type for a particular equipment brand. Majority of the experts interviewed have between 5 to 10 years and 15 to 20 years' experience in the subsea processing field. Aside the primary job function in table 3, all the respondents have engineering background with subsea fields in more than 5 geographic regions.

From the results, table 5 contains the FMEA results for helico-axial multiphase pump (HAP), table 6 for twin-screw pump (TSP) and table 7 for electrical submersible pump (ESP). It can be seen that each multiphase pump type has its area of application as some components that are still having issues in some equipment's (TSP and ESP) such as connector, cable, rotor are already proven in others (HAP). Part of the reason for this is that the pumps are of two different architecture, one is the rotodynamic pump in which has the helico-axial multiphase pump groups and the electrical submersible pump and the other is the positive displacement pump which has the twin-screw pump. Failure mode refers to the 
symptom of a failure and describes the effect by which a failure is observed. For the three multiphase pumps in table 5-7, the potential failure mode of their individual components is this failure of these components. Although the data is gathered from many sources, the most difficult source is from the equipment manufacturers as sometimes, some of their claims is refuted by the oil and gas producing companies who use these equipment's.

Table 1. Experts years of Experience

\begin{tabular}{|c|l|l|l|}
\hline Years of Experience & $5-10$ years & $15-20$ years & 20 years and above \\
\hline Total no of Experts & 3 & 3 & 1 \\
\hline
\end{tabular}

Table 2. Experts Academic Qualification

\begin{tabular}{|l|l|l|l|}
\hline Qualification & BSc & MSc & Ph.D \\
\hline Total no of Experts & 2 & 4 & 1 \\
\hline
\end{tabular}

Table 3. Experts Primary Job Function

\begin{tabular}{|l|l|l|l|}
\hline Job Function & Manager & Project Manager & Engineer \\
\hline Total no of Experts & 2 & 2 & 3 \\
\hline
\end{tabular}

Table 4. Experts Organization

\begin{tabular}{|l|l|l|l|}
\hline Organization & Oil and Gas Company & Engineering Contractor & Equipment Supplier \\
\hline Total no of Experts & 2 & 2 & 3 \\
\hline
\end{tabular}

Table 5. FMEA of Helico-Axial Multiphase Pump

\begin{tabular}{|c|c|c|c|c|c|c|}
\hline z & $\begin{array}{c}\text { Component \& } \\
\text { Function }\end{array}$ & $\begin{array}{c}\text { Potential } \\
\text { Failure } \\
\text { Mode }\end{array}$ & $\begin{array}{l}\text { Potential Effects of } \\
\text { Failure }\end{array}$ & $\begin{array}{l}\text { Potential } \\
\text { Causes of } \\
\text { Failure }\end{array}$ & $\begin{array}{c}\text { Current } \\
\text { Controls \& } \\
\text { Prevention }\end{array}$ & $\begin{array}{l}\text { Monitored } \\
\text { Parameters }\end{array}$ \\
\hline 1 & Motor & $\begin{array}{l}\text { Loss of } \\
\text { pump } \\
\text { function }\end{array}$ & Production failure & $\begin{array}{l}\text { Low lube oil } \\
\text { level } \\
\text { temperature / } \\
\text { low voltage }\end{array}$ & $\begin{array}{l}\text { Provide } \\
\text { adequate } \\
\text { cooling/monit } \\
\text { or high } \\
\text { temperatures }\end{array}$ & $\begin{array}{l}\text { Suction } \\
\text { pressure/ } \\
\text { /frequency }\end{array}$ \\
\hline 2 & $\begin{array}{l}\text { Mechanical } \\
\text { Seal }\end{array}$ & $\begin{array}{l}\text { Loss of } \\
\text { pump } \\
\text { function }\end{array}$ & $\begin{array}{l}\text { Elastomer } \\
\text { failure/Reduce } \\
\text { cooling/ equipment } \\
\text { failure (bearing \& } \\
\text { electric) }\end{array}$ & $\begin{array}{l}\text { High } \\
\text { temperature } \\
\text { from Low } \\
\text { lube oil level }\end{array}$ & $\begin{array}{l}\text { Appropriate } \\
\text { material } \\
\text { selection }\end{array}$ & $\begin{array}{l}\text { Discharge } \\
\text { pressure } \\
\text { /VSD output } \\
\text { power }\end{array}$ \\
\hline 3 & Impeller & $\begin{array}{l}\text { Loss of } \\
\text { pump } \\
\text { function }\end{array}$ & $\begin{array}{l}\text { Increase } \\
\text { temperature/Gas } \\
\text { Lock }\end{array}$ & $\begin{array}{l}\text { Gas volume } \\
\text { fraction } \\
\text { fluctuation }\end{array}$ & $\begin{array}{l}\text { Appropriate } \\
\text { material } \\
\text { selection }\end{array}$ & $\begin{array}{l}\text { Down hole } \\
\text { temperature } \\
\& \text { pressure }\end{array}$ \\
\hline 4 & Bearing & $\begin{array}{l}\text { Loss of } \\
\text { pump } \\
\text { function }\end{array}$ & $\begin{array}{l}\text { Bearing wear/ } \\
\text { Elastomer } \\
\text { failure/Reduce } \\
\text { cooling/decrease } \\
\text { head \& flow/affects } \\
\text { pump \& motor }\end{array}$ & $\begin{array}{l}\text { High } \\
\text { temperature } \\
\text { from Low } \\
\text { lube oil } \\
\text { level/decrease } \\
\text { d speed and } \\
\text { load }\end{array}$ & $\begin{array}{l}\text { Appropriate } \\
\text { lubrication } \\
\text { and material } \\
\text { selection }\end{array}$ & $\begin{array}{l}\text { Vibration } \\
\text { sensors/ } \\
\text { Wellhead } \\
\text { pressure \& } \\
\text { temperature/ } \\
\text { Lube oil } \\
\text { supply }\end{array}$ \\
\hline 5 & $\begin{array}{l}\text { Others } \\
\text { (pump,gear, } \\
\text { Driver shaft) }\end{array}$ & & & & & \\
\hline
\end{tabular}


Table 6. FMEA of Twin-Screw Pump

\begin{tabular}{|c|c|c|c|c|c|c|}
\hline z & $\begin{array}{l}\text { Compon } \\
\text { ent \& } \\
\text { Function }\end{array}$ & $\begin{array}{l}\text { Potentia } \\
1 \\
\text { Failure } \\
\text { Mode }\end{array}$ & $\begin{array}{l}\text { Potential Effects } \\
\text { of Failure }\end{array}$ & $\begin{array}{l}\text { Potential Causes of } \\
\text { Failure }\end{array}$ & $\begin{array}{l}\text { Current Controls \& } \\
\text { Prevention }\end{array}$ & $\begin{array}{l}\text { Monitore } \\
\mathrm{d} \\
\text { Paramete } \\
\text { rs }\end{array}$ \\
\hline 1 & Motor & $\begin{array}{l}\text { Loss of } \\
\text { pump } \\
\text { function }\end{array}$ & Motor wear & $\begin{array}{l}\text { increased speed/low } \\
\text { lubrication }\end{array}$ & $\begin{array}{l}\text { Adjust motor } \\
\text { windings }\end{array}$ & Flow rate \\
\hline 2 & $\begin{array}{l}\text { Mechani } \\
\text { cal Seal }\end{array}$ & $\begin{array}{l}\text { Loss of } \\
\text { pump } \\
\text { function }\end{array}$ & Seal failure & $\begin{array}{l}\text { High GVF/high } \\
\text { temperature/increas } \\
\text { ed speed/intermitted } \\
\text { gas bubbles }\end{array}$ & $\begin{array}{l}\text { Liquid recycling } \\
\text { system/ appropriate } \\
\text { material } \\
\text { selection/sand trap } \\
\text { installation } \\
\end{array}$ & $\begin{array}{l}\text { Differenti } \\
\text { al } \\
\text { pressure }\end{array}$ \\
\hline 3 & Rotor & $\begin{array}{l}\text { Loss of } \\
\text { pump } \\
\text { function }\end{array}$ & $\begin{array}{l}\text { Thermal } \\
\text { expansion of } \\
\text { rotors/screw } \\
\text { rubs/equipment } \\
\text { damage }\end{array}$ & $\begin{array}{l}\text { High gas volume } \\
\text { fraction/increased } \\
\text { pressure }\end{array}$ & & $\begin{array}{l}\text { Suction } \\
\text { pressure }\end{array}$ \\
\hline 4 & $\begin{array}{l}\text { Cooling } \\
\text { system }\end{array}$ & $\begin{array}{l}\text { Loss of } \\
\text { pump } \\
\text { function }\end{array}$ & $\begin{array}{l}\text { Severe damage } \\
\text { of the } \\
\text { equipment }\end{array}$ & Seal Oil pressure & $\begin{array}{l}\text { Adhering to the } \\
\text { recommended } \\
\text { practice }\end{array}$ & $\begin{array}{l}\text { al Shaft } \\
\text { power }\end{array}$ \\
\hline 5 & $\begin{array}{l}\text { Oil } \\
\text { refilling }\end{array}$ & $\begin{array}{l}\text { Loss of } \\
\text { pump } \\
\text { function }\end{array}$ & $\begin{array}{l}\text { Severe damage } \\
\text { of the } \\
\text { equipment }\end{array}$ & Seal Oil pressure & $\begin{array}{l}\text { Adhering to the } \\
\text { recommended } \\
\text { practice }\end{array}$ & GVF \\
\hline 6 & Bearing & $\begin{array}{l}\text { Loss of } \\
\text { pump } \\
\text { function }\end{array}$ & $\begin{array}{l}\text { Bearing } \\
\text { damage }\end{array}$ & $\begin{array}{l}\text { increased } \\
\text { speed/mechanical } \\
\text { seal leakage }\end{array}$ & $\begin{array}{l}\text { Improved } \\
\text { materials/tolerances/ } \\
\text { control vibration }\end{array}$ & $\begin{array}{l}\text { alTempera } \\
\text { ture }\end{array}$ \\
\hline 7 & $\begin{array}{l}\text { Others } \\
\text { (Pumps, } \\
\text { gear, } \\
\text { screw, } \\
\text { shafts ) }\end{array}$ & $\begin{array}{l}\text { Loss of } \\
\text { pump } \\
\text { function }\end{array}$ & $\begin{array}{l}\text { Increase } \\
\text { temperature/Los } \\
\text { s of volumetric } \\
\text { efficiency/corro } \\
\text { ded gear }\end{array}$ & $\begin{array}{l}\text { High GVF/ } \\
\text { differential } \\
\text { pressure/ } \\
\text { /mechanical seal } \\
\text { leakage }\end{array}$ & $\begin{array}{l}\text { Improved } \\
\text { materials/tolerances }\end{array}$ & \\
\hline
\end{tabular}

Table 7. FMEA of Electrical Submersible Pump

\begin{tabular}{|l|l|l|l|l|l|l|}
\hline Z & $\begin{array}{l}\text { Compon } \\
\text { ent \& } \\
\text { Function }\end{array}$ & $\begin{array}{l}\text { Potential } \\
\text { Failure } \\
\text { Mode }\end{array}$ & $\begin{array}{l}\text { Potential } \\
\text { Effects of } \\
\text { Failure }\end{array}$ & $\begin{array}{l}\text { Potential Causes of } \\
\text { Failure }\end{array}$ & $\begin{array}{l}\text { Current } \\
\text { Controls \& } \\
\text { Prevention }\end{array}$ & $\begin{array}{l}\text { Monito } \\
\text { red } \\
\text { Parame } \\
\text { ters }\end{array}$ \\
\hline 1 & $\begin{array}{l}\text { Electrica } \\
\text { f failure } \\
\text { in Motor }\end{array}$ & $\begin{array}{l}\text { Loss of } \\
\text { pump } \\
\text { function }\end{array}$ & Motor wear & $\begin{array}{l}\text { High temperature/voltage } \\
\text { and other operating } \\
\text { conditions }\end{array}$ & $\begin{array}{l}\text { Change motor } \\
\text { windings }\end{array}$ & $\begin{array}{l}\text { viscosit } \\
\text { y }\end{array}$ \\
\hline 2 & $\begin{array}{l}\text { Mechani } \\
\text { cal Seal }\end{array}$ & $\begin{array}{l}\text { Loss of } \\
\text { pump } \\
\text { function }\end{array}$ & $\begin{array}{l}\text { Mechanical } \\
\text { seal wear }\end{array}$ & $\begin{array}{l}\text { High temperature / } \\
\text { pressures and corrosive } \\
\text { chemicals }\end{array}$ & $\begin{array}{l}\text { Use of } \\
\text { improved } \\
\text { materials }\end{array}$ & $\begin{array}{l}\text { ESP } \\
\text { Freque } \\
\text { ncy }\end{array}$ \\
\hline 3 & $\begin{array}{l}\text { Connect } \\
\text { or }\end{array}$ & $\begin{array}{l}\text { Reduced } \\
\text { pump } \\
\text { performance }\end{array}$ & $\begin{array}{l}\text { Elastomer } \\
\text { degradation/ } \\
\text { penetrators }\end{array}$ & $\begin{array}{l}\text { High temperature/high } \\
\text { pressures }\end{array}$ & $\begin{array}{l}\text { Appropriate } \\
\text { pressure gauges }\end{array}$ & $\begin{array}{l}\text { Densi } \\
\text { ty }\end{array}$ \\
\hline 4 & Cable & $\begin{array}{l}\text { Loss of } \\
\text { pump } \\
\text { function }\end{array}$ & Cable wear & $\begin{array}{l}\text { temperature \& pressure } \\
\text { variation/ high voltage } \\
\text { and corrosive chemicals }\end{array}$ & $\begin{array}{l}\text { Proper } \\
\text { engineering } \\
\text { design }\end{array}$ & $\begin{array}{l}\text { Flow } \\
\text { rate }\end{array}$ \\
\hline 5 & Impeller & $\begin{array}{l}\text { Reduced } \\
\text { pump } \\
\text { performance }\end{array}$ & Gas Lock & $\begin{array}{l}\text { Gas volume fraction/ } \\
\text { Temperature }\end{array}$ & $\begin{array}{l}\text { Installing mixer } \\
\text { upstream/use of } \\
\text { demulsifier and } \\
\text { scale inhibitors }\end{array}$ & GVF \\
\hline
\end{tabular}




\begin{tabular}{|l|l|l|l|l|l|l|}
\hline 6 & Bearing & $\begin{array}{l}\text { Loss of } \\
\text { pump } \\
\text { function }\end{array}$ & $\begin{array}{l}\text { Bearing } \\
\text { wear }\end{array}$ & $\begin{array}{l}\text { Improved } \\
\text { materials/tolerances/contr } \\
\text { ol vibration }\end{array}$ & $\begin{array}{l}\text { Reduce } \\
\text { vibration }\end{array}$ & \\
\hline 7 & $\begin{array}{l}\text { Others } \\
\text { (Pumps, } \\
\text { shaft, } \\
\text { diffuser, } \\
\text { thrust) }\end{array}$ & & & & $\begin{array}{l}\text { Stabilizers to } \\
\text { eliminate shaft } \\
\text { failure/ diffuser } \\
\text { coated with } \\
\text { Teflon }\end{array}$ & \\
\hline
\end{tabular}

\section{Conclusions}

The purpose of the FMEA is to identify the critical components and their failure modes and effects which serve as an input towards a process of modeling and predicting failures of these equipment's to increase the reliability performance which is especially needful because of the environment these equipment's will be deployed in. The components are important as they form the building block that specifies the equipment reliability. Using the FMEA as a guide, a lot can be achieved in understanding the overall system architecture.

\section{References}

1. B.D. Gould, OTC, (2011)

2. F.A. Albuquerque, M.G. Morais, M.L. Euphemio, C. Kuchpil, D.G. Duarte, R.T. Orlowski. OTC Brasil, (2013)

3. D. Ishita, NTNU, (Unpublished Masters Thesis) (2012)

4. F.J.S. Alhanati, F.E.Trevisan, OTC, (2012).

5. V.F. Teixeira, T.R. Gessner, I.T. Shigueoka, SPE Latin American Petro Engrg Conf (2012)

6. G. Hua, G. Falcone, C. Teodoriu, G.L. Morrison, Oil and Gas Facilities, 36-46, (2012)

7. J. Zhang, H. Zhu, H. Wei, J. Peng, Appl. Mech. \& Mats, vols 52-54, 399-404 (2011)

8. J. Zhang, H. Zhu, H. Wei, Z. Li, L. Xiong, Int. Conf. on Intel. Compu. Tech. \& Automation. (2010)

9. A. Williams, Urban B., Margulis J., SPE Annu. Tech. Conf. \& Exhib., (2010)

10. K. Räbiger, T.M.A. Maksoud, J. Ward, G. Hausmann. Exp. Thermal \& Fluid Sci. 32, 1694-1701, (2008)

11.D. Ing, M. Reichwage, (2008), http://www.german-oilgas-expo.com/Bornemann-CMMultiphase.pdf

12. T. Gao, D. Yang, F. Cao, J. Jiao. Appl. Phys. Engrg, 12 (9), 720-730, (2011) 